\title{
APPLICATIONS OF COMPUTER VISION IN AGRICULTURE
}

\author{
Pranav Ghadge \\ School of Computer Engineering and Technology \\ Dr. Vishwanath Karad MIT World Peace University, \\ Pune, Maharashtra, India \\ Prasanna Sand \\ School of Computer Engineering and Technology \\ Dr. Vishwanath Karad MIT World Peace University, \\ Pune, Maharashtra, India
}

\begin{abstract}
Satellite system advances, remote sensing and drone technology are continuing. These progresses produce high-quality images that need efficient processing for smart agricultural applications. These possibilities to merge computer vision and artificial intelligence in agriculture are exploited with recent deep educational technology. This involves essential phenomena of data and huge quantities of data stored, analysed and used when making decisions. This paper demonstrates how computer vision in agriculture can be used.
\end{abstract}

\section{Keywords- Computer Vision, Agriculture.}

\section{INTRODUCTION}

Humans look at and see the world around them visually through their eyes and minds. The science of computer vision is intended to give a system or computer the same, if not better power. Computer vision requires the automated retrieval, interpretation and comprehension of useful and significant information from a single frame or image set. In order to achieve an automated visual perception, a logically and algorithmically dependent is involved. Computer vision, which involves a less evaluative methodology, has the ability to estimate the characteristics of food with the advantage of accelerated speed, ease of use and limited preparation for samples for training. Computer vision systems are especially feasible to classify food products into particular grades, detect deficiencies and estimate properties like colour, structure, scale, surface defects and contamination.

\section{LITERATURE SURVEY}

The paper [1] develops an algorithm for detecting and preventing the spreading of diseases to the whole crop and results in high quality crop production.

\author{
Riddhik Tilawat \\ School of Computer Engineering and Technology \\ Dr. Vishwanath Karad MIT World Peace University, \\ Pune, Maharashtra, India \\ Parul Jadhav \\ School of Electronics and Communication Engineering \\ Dr. Vishwanath Karad MIT World Peace University, \\ Pune, Maharashtra, India
}

The proposed system in [2] aims to develop a computer vision based robotic weed control system (WCS) for real-time control of weeds in onion fields. This system will be able to identify weeds and selectively spray right amount of the herbicide

They [3] developed a low-cost automated drought detection system using computer vision coupled with machine learning (ML) algorithms that document the drought response in corn and soybeans field crops. Using ML, we predict the drought status of crop plants with more than $80 \%$ accuracy relative to expert-derived visual drought ratings.

In paper [4] they presented Agriculture-Vision: a large-scale aerial farmland image dataset for semantic segmentation of agricultural patterns. We collected 94, 986 high-quality aerial images from 3, 432 farmlands across the US, where each image consists of RGB and Near-infrared (NIR) channels with resolution as high as $10 \mathrm{~cm}$ per pixel.

In paper [5] a computer vision algorithm runs on the backbone of the Internet of Leaf Things (IoLT) based gCrop system to calculate the growth patterns of the leaves in realtime. The model shows a great potential with an accuracy of around $98 \%$ to predict the growth of the leaves. Thus, it is promisingly expected that this system will effectively contribute in strengthening the current farming practices by ensuring the quality of the crops and improving the production yield.

This paper [6] aims to give insights on the integration of computer vision for smart farming in-order to attain sustainable agriculture. Using a structured approach, this research proposes a computer vision technique for crop image feature characterization that applies in the determination of the crop's health status. 


\section{APPLICATIONS}

\section{Sorting \& Classifying of Vegetables \& Fruits.}

Latest methods, like deep learning, are able to complete with humans in conventional vision algorithms. If a set of "good" fruit (such as oranges) and a number of oranges with defections are presented in the deep learning algorithm, additional oranges based on the sample sets are automatically categorised (graded). There is no need for fine tuning. An orange is categorised and graded accordingly each time that it looks like one of the sets. This is a quick and effective method; it provides consistent results, more importantly. The profound learning is the most modern approach for many applications of this kind that we recommend today

\section{Image processing for Agriculture}

We can reduce the cost beared by the farmers by using computer vison algorithms. To detect fruit diseases, we can apply Machine learning to the photos taken with cell phones, enabling a timely intervention; real-time weed detection by tractors can allow herbicides to be applied and chemicals to be used less frequently; water leakage can be detected by infrared cameras in large fields. Cameras mounted above conveyor belts can be used as a production line for the realtime sorting and grading of agricultural products, cameras in to track plant growth in greenhouses and to control animals' action camera can be used.

Due to the complexity in farming and outdoor weather conditions a rise can be expected. In sophistication of CV algorithms. There are no off-the-shelf algorithms that can account for the enormous variability encountered in real applications in weather, soil and other environmental conditions. Computer vision and machine learning researchers therefore have been developing algorithms to meet the demanding conditions both outdoors and indoors, as well as meet the exact requirements of our agricultural customers.

\section{Phenotyping}

In this area, phenotyping relies mainly on advanced computer vision algorithms. As plants often cultivate in unregulated environments and yet precision farming requires precision measurements by its very nature, a combination of algorithm types is better suited to phenotyping projects, which will provide precise and robust measurements. At least one camera for 1 to 3 plants to be analyzed/detected automatically consists of one single instance of the device. The key characteristics of interest include the height and width of the plant in regions, colour analysis and automatic estimate of fruit yield.
The first challenge, as in most outdoor situations, was to detect the target plant and separate it from the atmosphere or the rest of the plants. Data is pre-processed in order to eliminate unnecessary portions of the picture and yet other conditions and plant types can be generalised.

In three steps, plant detection was developed. The initial step produces the general location of the facility and is achieved in a relatively low resolution based on colour index and structural ques, saving computational resources and power. The next step will be the accurate identification of the plant, using an iterative process that combines short segments to achieve texture or geometry anomalies, which reflect the change between the environment and the plant. The segmentation is then further refined, taking previous knowledge of the plant and the environment into account. The next step is to extract plant measurements with different techniques, based on various colour indices and textures. Measuring is then converted to an important unit of measurement from pixel units (e.g. cm). In two ways, such a transformation can be generated:

(i) The plant may be placed next to an artificial reference object, which provides an anchor to accurately calculate the transformations needed; or

(ii) the device hardware can be integrated with sensors that allow depth estimation, such as Stereo Cameras, removing the need for a repository object. Upon identification and good estimation of the position of the plant, implementation of more layers of algorithm can be done: detection of plant structures, such as the flowers and fruits, and to calculate their yield, dimensions and colour can be done with modern machine learning systems.

\section{Plant Diseases}

To reduce broad surveillance work on big crop farms automated techniques are helpful to detect plant Diseases, it also detects disease symptoms very early. For automatic detection, as well as for classification of leaf diseases and survey of different disease classification techniques which could be used for plant leaf disease recognition, an algorithm may be used for the image segmentation technology. The segmentation of images is carried out by genetic algorithm and is an important factor for detecting disease in plant leaf disorders.

\section{CONCLUSION}

In general, computer vision technology implementations are discussed in this paper on the basis of review studies in the past few years and analysed in depth in the field of agricultural automation. In particular, the paper focuses on four areas: plant diseases, phenotyping, fruit and vegetables 
grading and sorting and agriculture precision. We may deduce that prior work contributes to the development in individual fields of agricultural automation, with the welfare of low cost, high productivity and high precision. further, we studied the problems posed by computer vision technology in potential farm automation applications centred on the status quo. Firstly, as the technology continues to evolve in the future, it is important to create a broad dataset in order to achieve flexibility and coordination of the technology. The technology and problems that must be faced in the future will at the same time continue to evolve. Secondly, with the rapid growth of the field of agricultural automation, more disciplines will be included and the quality and quantity demand of professionals will continue to increase. Finally, it will also be difficult to ensure accuracy and strength of relevant technologies in various complex circumstances, given the complex environmental context of agricultural production management.

On the basis of the above review and discussion, we conclude that computer vision technology would be best used in agriculture as an emerging technology. Computer vision information technology will in future be commonly used for all aspects of farm production management based upon largescale data sets and will be used in larger numbers to solve the present agricultural problems. Computer vision technology and artificial intelligence algorithms can enhance economical efficiency, general performance, co-ordination and robust agricultural automation systems performance. Agricultural automation equipment and systems can be built more intelligently by implementing cutting-edge technologies such as deep learning technologies and spectral analysis technology. In future, the productivity and quality of agricultural production will be enhanced with the implementation and advancement of computer vision technologies, providing useful recommendations and insights for farmers in the field of support for decision-making and action and timely and systematic development of agricultural automation.

\section{REFERENCES}

[1] Kuricheti G. and Supriya P., (2019), "Computer Vision Based Turmeric Leaf Disease Detection and Classification: A Step to Smart Agriculture," 2019 3rd International Conference on Trends in Electronics and Informatics (ICOEI), Tirunelveli, India, 2019, pp. 545-549.

[2] Arakeri M. P., Vijaya Kumar B. P., Barsaiya S. and Sairam H. V., (2017) "Computer vision based robotic weed control system for precision agriculture," 2017 International Conference on Advances in Computing, Communications and Informatics (ICACCI), Udupi, 2017, pp. 1201-1205, doi: 10.1109/ICACCI.2017.8126005.
[3] Ramos-Giraldo P., Reberg-Horton C., Locke A. M., Mirsky S. and Lobaton E., (2020) "Drought Stress Detection Using Low-Cost Computer Vision Systems and Machine Learning Techniques," in IT Professional, vol. 22, no. 3, pp. 27-29, 1 May-June 2020, doi: 10.1109/MITP.2020.2986103.

[4] Chiu M. T. et al., (2020) "Agriculture-Vision: A Large Aerial Image Database for Agricultural Pattern Analysis," 2020 IEEE/CVF Conference on Computer Vision and Pattern Recognition (CVPR), Seattle, WA, USA, pp. 2825-2835, doi: 10.1109/CVPR42600.2020.00290.

[5] Kumar S., Chowdhary G., Udutalapally V., Das D. and Mohanty S. P., (2019) "gCrop: Internet-of-Leaf-Things (IoLT) for Monitoring of the Growth of Crops in Smart Agriculture," 2019 IEEE International Symposium on Smart Electronic Systems (iSES) (Formerly iNiS), Rourkela, India, 2019, pp. 53-56, doi: 10.1109/iSES47678.2019.00024.

[6] TOMBE R., (2020) "Computer Vision for Smart Farming and Sustainable Agriculture," 2020 IST-Africa Conference (IST-Africa), Kampala, Uganda, 2020, pp. 1-8. 